\title{
EXPLORE AND COMPARE THE KNOWLEDGE, ATTITUDE, AND
ACCEPTANCE OF DENTAL IMPLANT AS A TREATMENT OPTION AMONG
SUB-POPULATION OF SAUDI ARABIA
}

\section{ABSTRACT}

Objective: Implant therapy has gained wide popularity in the recent years. A complete information on implant therapy should be provided to patient by the dentist. The aim of present study is to evaluate the knowledge, attitude, source of information and acceptance of dental implants among the general population in the Southern region of Saudi Arabia.

Materials and Methods: A total of 712 patients were randomly selected from the OPD of dental hospitals across the southern region of Saudi Arabia. The designed questionnaire comprised of 15 open-ended questions. Only Saudi national population was selected for the survey. The questionnaire was divided into demographic data, knowledge, attitude and participant's acceptance towards implant therapy. The collected data were statistically analyzed using the Statistical Package for Social Sciences.

Results: Out of 712 participants, 675 complete the survey. $59.1 \%$ were male and $41.9 \%$ were females with an average age of 39.6 years old. All variables except for age showed statistically significant difference in the knowledge about implant among the study groups $(\mathrm{p}<0.05)$. The knowledge score was directly related to education and professional status of the participants. Majority of the patients $(66 \%)$ with a history of implant therapy were satisfied with the placed implant. $41 \%$ of the participants reported high cost as a limiting factor for selecting an implant treatment.

Conclusions: Population from the southern region of Saudi Arabia has moderate knowledge about dental implant treatment. Dentists were found to be the most important source of information to the patient. The major barrier for not selecting implant treatment was the high cost of the implant therapy.
D* Shahabe Saquib Abullais ${ }^{1}$ (iD) Nabeeh Abdullah Al-Qahtani ${ }^{1}$ (i) Ahmed Al-Ahmeri ${ }^{1}$ (D) Saad Al-Qahtani ${ }^{1}$ (D)Mohammed Yunis Saleem Bhatt ${ }^{1}$ (D)Muhammed Abid Khan ${ }^{2}$

ORCID IDs of the authors: S.S.A. 0000-0002-5223-2167 N.A.AQ. 0000-0001- 5064-7556 A.AA. 0000-0002-4770-5281 S.AO. 0000-0003-4724-4021 M.Y.S.B. 0000-0002-6931-9381

M.A.K. 0000-0001-5977-3989 ${ }^{1}$ Department of Periodontics and Community
Dental Sciences, College of Dentistry, King
Khalid University, Abha, Saudi Arabia
2 College of Medicine, King Khalid
University, Abha, Saudi Arabia

Keywords: Implant, knowledge, acceptance, attitude, Saudi Arabia.

How to Cite: Abullais SS, Al-Qahtani NA, Al-Ahmeri A, Al-Qahtani S, Bhatt MYS, Khan MA. Explore and Compare the Knowledge, Attitude, and Acceptance of Dental Implant as A Treatment Option Among Sub-Population of Saudi Arabia. Cumhuriyet Dent J 2019;22:2:241-248.

*Corresponding Author:

Department of periodontics and community dental sciences, King Khalid University, College of Dentistry, Abha, KSA.

Phone: +966583056343 Facsimile numbers E-mail: drsaquin24@gmail.com 


\section{INTRODUCTION}

The objective of advanced dental practice is to restore the patient to normal function, aesthetics, and phonetics. ${ }^{1}$ The increasing use of dental implants is an attempt to fulfill these objectives in dental practice since last few decades. ${ }^{2}$ In the recent years dental implants has gained popularity within the dental community and extensively recognized as a prosthetic treatment option of complete or partially edentulous and completely patients. ${ }^{3}$ Many longitudinal studies have confirmed the long-term success and survival of dental implant as a prosthetic option, which has increased the prevalence of dental implant treatment exponentially. ${ }^{4}$ Most of the patients treated by dental implants have revealed improvement in the quality of lifestyle and confidence.

Studies in recent years have revealed that the acceptance of implant therapy is increasing in both partially edentulous and completely edentulous patients. Nearby et al has conducted a study in Sweden in two parts over the course of ten years. At the time of the first survey in 1989, about $32 \%$ of participants stated a desire for implant therapy, which was dramatically increased to $95 \%$ in the second survey i.e in $1999 .{ }^{5,6}$ A study on patient's attitude after dental implant therapy revealed that the majority of patients were ready to accept implant treatment again if needed and their oral health and selfconfidence had increased significantly. ${ }^{7}$ Another study conducted among Saudi population showed that about $76 \%$ willing to undergo the same treatment again and $79 \%$ would recommend it to the others. ${ }^{8}$

Many surveys are available in the literature, to evaluate a patient's awareness of dental implants therapy. The level of awareness and knowledge is varied among several surveys conducted across different countries. A survey conducted by Zimmer et al, in the American population, revealed a high awareness rate and positive attitude towards oral implant treatment. participants for the survey also agreed that esthetic results are better with implant therapy. ${ }^{9} \mathrm{~A}$ survey conducted on 379 participants in Riyadh, Saudi Arabia revealed that $66.4 \%$ of the participants were aware of the dental implants as a treatment option. ${ }^{10}$ Another study conducted in Malaysian population showed that, among 1013 participants, $772(76 \%)$ were aware of dental implant as a treatment option. ${ }^{11} \mathrm{~A}$ study conducted in Finland showed that, the level of awareness of implant treatment among selected groups was $29 \%^{12}$, whereas survey from Australia showed a higher level of awareness of about $64 \% .^{13}$

Implant therapy is an elective procedure. Complete information on implant therapy and all other treatment options should be provided to the patient so that they can make a decision to choose the most appropriate option. ${ }^{14} \mathrm{~A}$ survey on the Australian population disclosed that dentists are the principal source of information on dental implants to the patients followed by friends and print media and the general practitioners. Also, of those interested in gaining more information regarding implants, a majority of them wanted it from the dentists. ${ }^{15}$ In another nationwide survey of the Indian population, it was concluded that information about dental implants was mainly provided by the dental surgeon. ${ }^{16}$ Results from the other studies showed that the subject's friends and their relatives were the main sources of information about dental implants, whereas dentists were the secondary source of information. ${ }^{10,11}$

To the best of author's knowledge, there is no existing literature regarding the patient's knowledge, awareness about dental implants in the Southern region of Saudi Arabia. Thereforethe aim and objectives of the present study are to1) evaluate the knowledge regarding dental implants among the selected populations-

2) evaluate the sources of information concerning the dental implant as a treatment option-

3) evaluate the level of acceptance of dental implants as a treatment option.

\section{MATERIALS AND METHODS}

The present one-point, cross-sectional survey was conducted from 15th October 2016 to 26th February 2017. A total of 712 patients were randomly selected from the outpatient department of various government dental hospitals. A selfexplanatory standardized questionnaire 
comprising of 15 questions was designed to assess and compare patient's knowledge, acceptance and attitude towards dental implants in the Southern region of Saudi Arabia.

The questionnaire comprised of four main domains: 1)Demography-age, occupation and educational status of the participants. 2)Knowledge of the participants towards dental implant treatment. 3)The attitude of the participants towards implant therapy- 4)Participant's acceptance of implant therapy. A pilot test was performed on 15 participants to evaluate the efficacy of the questionnaire. The questionnaire was prepared in English as well as Arabic language considering the population in the area. Only Saudi national population was selected for the survey while other nationalities were excluded from participating.

The sample size was calculated depending on the following formula; ${ }^{12}$

$$
\frac{\frac{Z^{2} \cdot p(1-p)}{\mathrm{e}^{2}}}{1+\left(\frac{Z^{2} \cdot p(1-p)}{\mathrm{e}^{2} N}\right)}
$$

Where:

$Z=Z$ value (1.96 for $95 \%$ confidence level)

$p=$ percentage of picking a choice expressed as a decimal (0.5)

This was found to be $50 \%$ for the present study which was expressed as 0.50 .

$\mathrm{e}=$ confidence interval, expressed as decimal $(0.05)$

$\mathrm{N}=$ total population of the region

By using the above formula- the minimum sample size calculated was 384 .
The study protocol was presented to the Institutional Ethics Committee (IEC) and ethical clearance was obtained from them. The importance of the study was explained verbally to the participants and written informed consent was obtained before completion of the questionnaire form. The questionnaire did not contain the name of the participants; thus the confidentiality of the participants was maintained. The questionnaires were distributed among the participants during their hospital visits, and the research team members coordinates were around to answer any queries related to the questionnaire.

All the hypotheses were formulated using two-tailed alternatives against each null hypothesis (hypothesis of no difference). The entire data was statistically analyzed using the Statistical Package for Social Sciences (SPSS)version 21.0- (IBM Corporation, USA) for MS Windows. Chi-square test was executed to compare the descriptive data. $\mathrm{P}$ value- $<0.05$ was taken as statistically significant.

\section{RESULTS}

Out of 712 participants, 685 completed the survey with a response rate of $(96.20 \%)$. Among the 685 responses received, 10 were incomplete and thus excluded from the survey. Therefore 675 complete responses were selected for the final statistical analysis. Table 1 provides a detailed summary about the demographic data of the surveyed individuals. Out of 675 participants, $59.1 \%$ were males and $41.9 \%$ were females with an average age of 39.6 years old. Majority of the participants $(48 \%)$ belonged to a young adult category. Figure 1 presents the distribution of overall knowledge of the surveyed participants. 
Table 1. Demographic distribution of the participants.

\section{Gender}

Male

Female

Age

Adolescence (10-19)

Young Adult (20-35)

Middle Age Adult(36-49)

Old Age Adult (50-onwards )

Education

Primary School

Secondary School

Bachelor degree

Master degree

Occupation

Students

Housewife / Unemployed

School / University Teacher

Professionals

Business

Government Employee

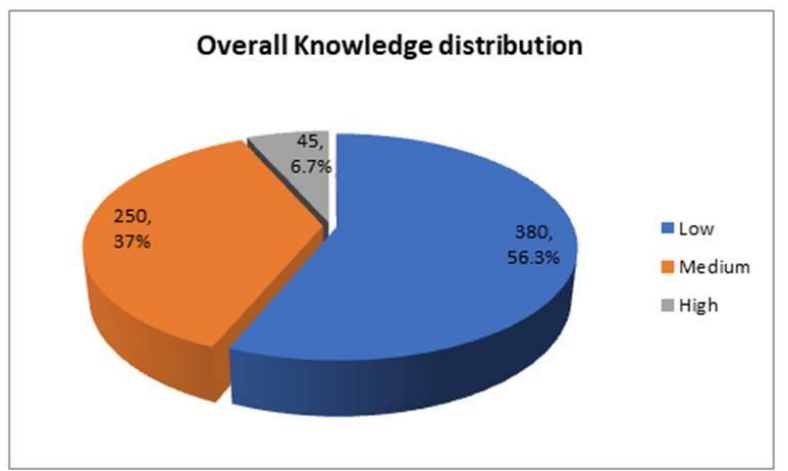

Figure 1. Overall distribution of knowledge among the participants.

In response to the question about alternatives to replace missing teeth, $94.8 \%$ of the participants were aware about one of the modality used for the replacement of missing teeth. Majority of them were aware about removable and fixed partial denture. For the question about advantages of fixed over removable prosthesis, $60.4 \%$ of the participants opted that fixed prosthesis has more esthetic outcome and they resembles natural teeth. Out of all participants, $69.33 \%$ had heard about

\section{Frequency (n)}

Percentage (\%)

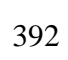

283

58.1

41.9

140

20.7

$324 \quad 48.0$

$189-28.0$

$22 \quad 3.3$

$42 \quad 6.2$

$284 \quad 42.1$

$302 \quad 44.7$

$47-7.0$

$219 \quad 32.4$

$137 \quad 20.3$

$115 \quad 17.0$

$\begin{array}{ll}59 & 8.7\end{array}$

$29 \quad 4.3$

$115 \quad 17.0$

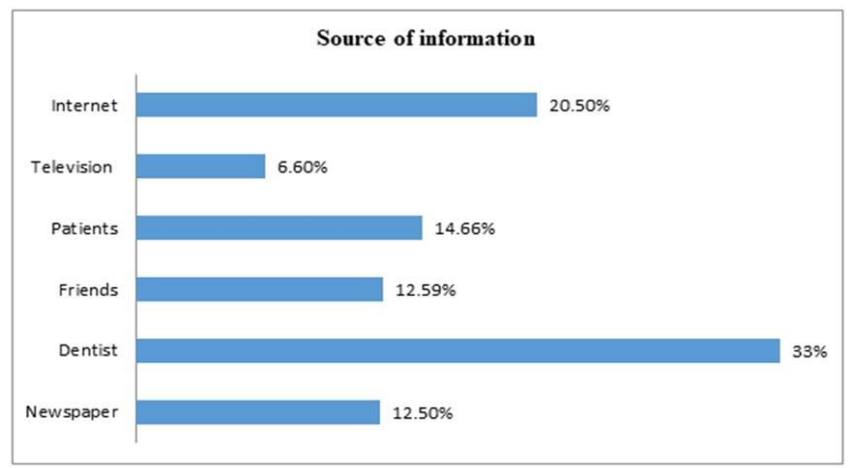

Figure 2. Distribution of source of information related to implant therapy among the participants.

the dental implant terminology, and they were aware of it as a treatment option. The dentists were the main source of information about dental implants in $31.5 \%$ of the questioned participantsfollowed by internet in $20.5 \%$ and patients in $14.6 \%$ (Figure 2).

Table 2 represents a detailed distribution and comparison of knowledge with respect to gender, age, education, and occupation. 
Table 2. Distribution and comparison of knowledge category with respect to different variables among the participants

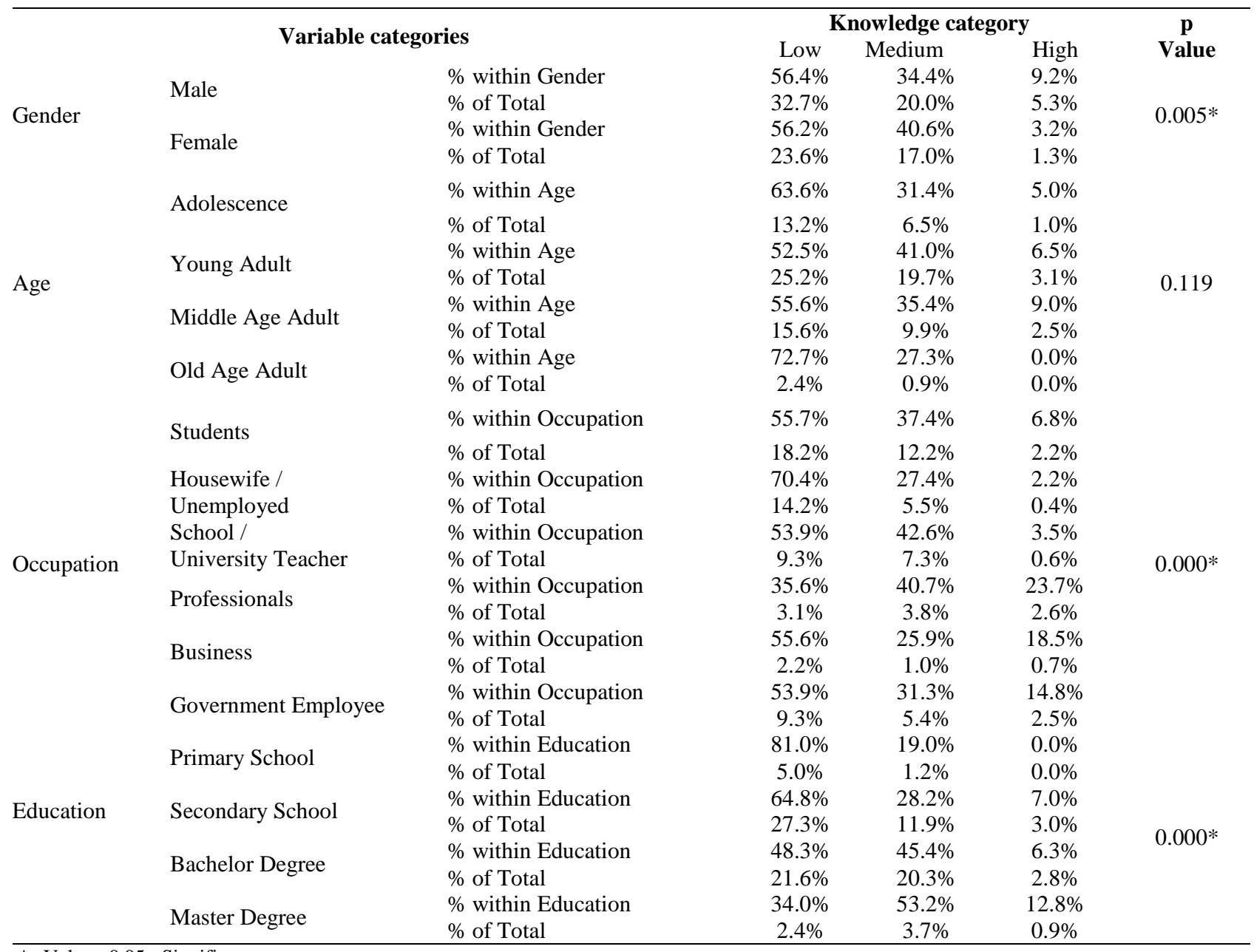

${ }^{*} p$ Value $<0.05=$ Significant

Knowledge was divided into 3 categories; low, medium and high- depending on the percentage of correct response to the questions. Knowledge was considered as high, medium or poor, if the correct response selected by respondents were more than $70 \%$, between $50-70 \%$ and less than $50 \%$ respectively. All the variables except for age showed- statistically significant difference in the knowledge about implant among the study participants $(p<0.05)$. When asked about the previous exposure to implant therapy, $19.6 \%$ of the participants provided a positive response. Out of 123 participants who have received implant therapy in the past, $66 \%$ were satisfied, $26 \%$ were partially satisfied and $8 \%$ were unsatisfied with the results of therapy (Figure-3). Figure 4 shows the detailed distribution of the perception among the participants towards dental implant therapy.

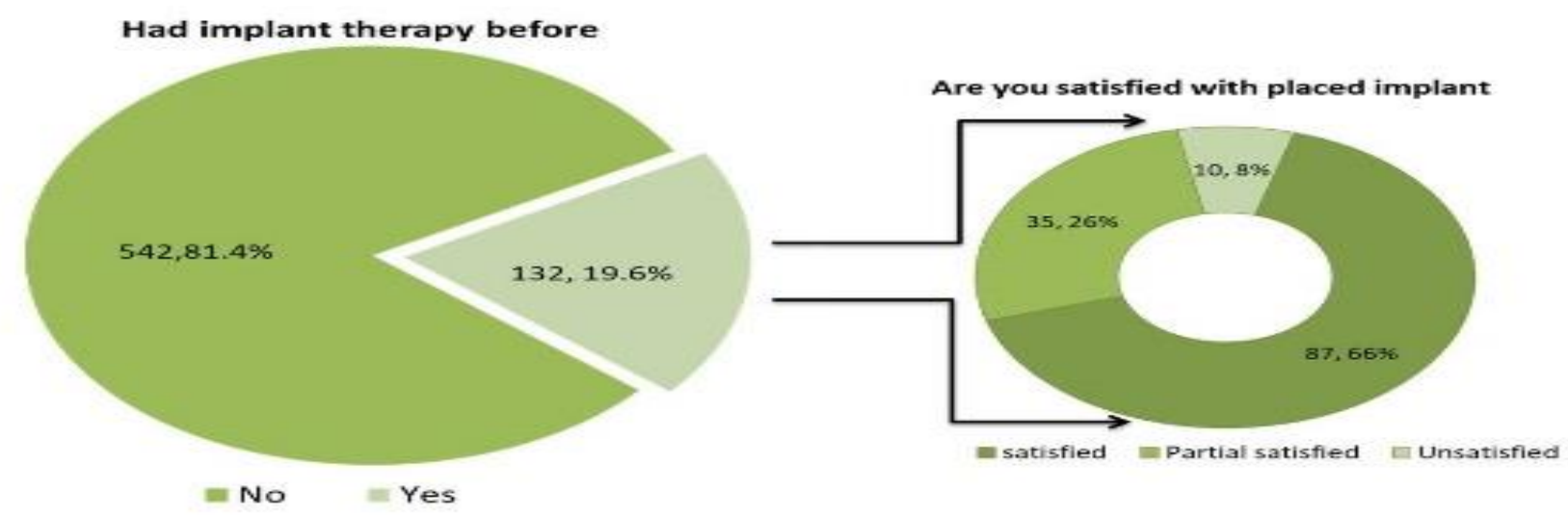

Figure 3. Acceptance and satisfaction related to implant therapy among participants. 


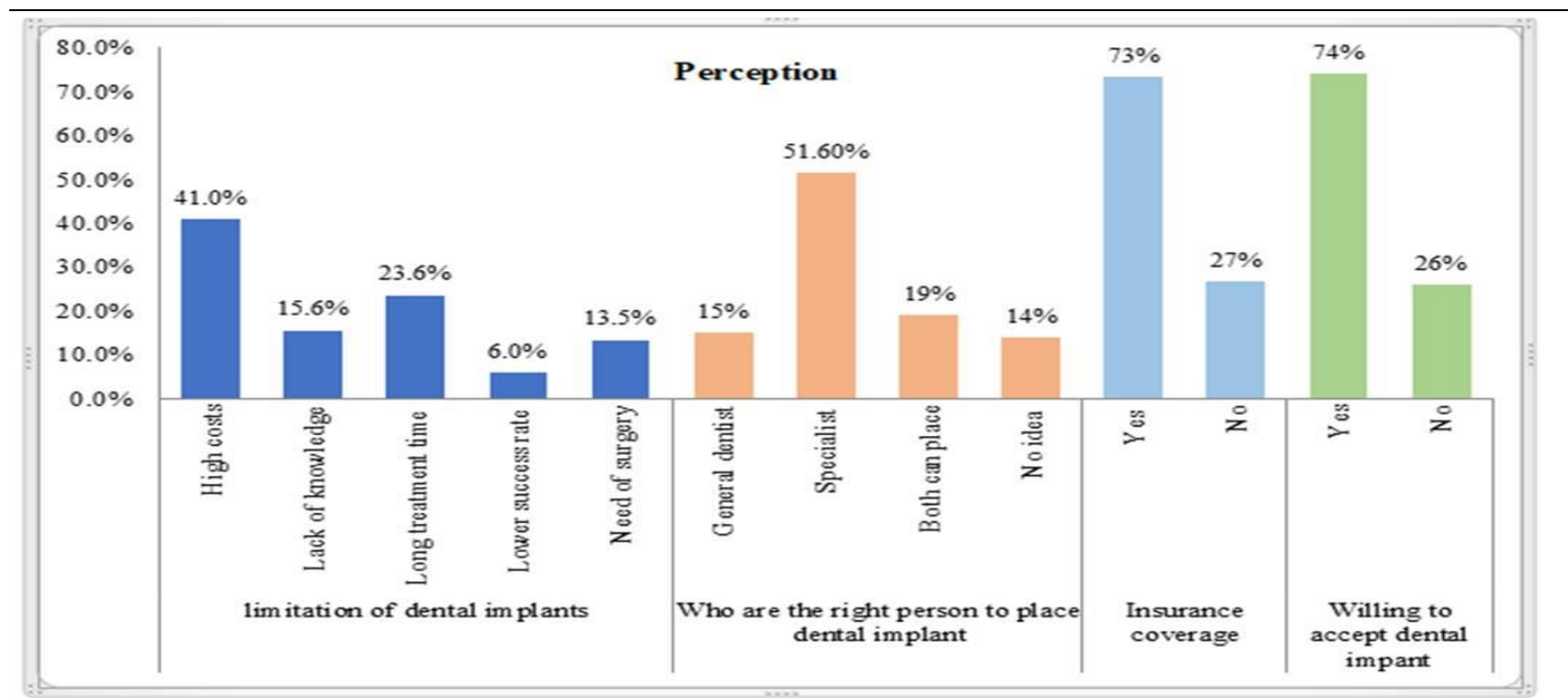

Figure 4. Distribution of participants' perception regarding dental implant therapy.

\section{DISCUSSION}

In the recent years dental practice has changed enormously due to the incorporation of dental implant as a treatment option. Dental implant is becoming a popular treatment modality in the general population with a long-term of success and higher patient satisfaction. In the last few years- a dental implant is gaining popularity and acceptance in the Southern region of Saudi Arabia. To the best of author's knowledge, this is the first survey conducted in the Southern region of Saudi Arabia to evaluate the knowledge, perception, and acceptance of dental implant.

In the present study, $69.33 \%$ of the participants were aware of the dental implant as a treatment modality. This findings is in accordance with the other studies conducted by Shivani et al..$^{11}$ - Tepper et al. ${ }^{17}$, and Sulieman et al. ${ }^{10}$, which reported awareness about $76.2 \%, 72 \%$, and $66.4 \%$ - respectively. A study conducted by Ceyda et $a l .{ }^{18}$, in the Turkish population had contradicting results, with an awareness of only $45 \%$. Most of the patients from the study group felt that fixed prosthesis is better and appears more natural than a removable prosthesis. These findings are in consistent with the study done by Tepper et al. ${ }^{17}$, and Ceyda et al. ${ }^{18}$ In the present survey- the major source of information dentists (33\%)- followed by internet $(20.5 \%)$ and patients (14.66\%). The present information sources resemble those reported by Ceyda et al. ${ }^{18}$, and Pommer et al. ${ }^{15}$ However, Zimmer et al. ${ }^{9}$, reported news media and Sulieman et al. ${ }^{10}$, reported friends and their relatives as the main source of information.

The knowledge of the participants was evaluated by asking six questions with a score range of 0-6. Knowledge was considered as high if correct answers were more than $70 \%$, medium if between $50-70 \%$ and poor if less than $50 \%$. The results of the survey showed that the difference in the knowledge between gender was statistically significant $(\mathrm{p}<0.005)$, males reported higher knowledge score than females. While considering age as a variable, there was no statistically significant difference among the different age group ( $\mathrm{p}>0.119$ ), but young age adults showed better knowledge score compared to other age groups. This can be related to the fact that the younger generation has more interest in dental implant treatment. A significant difference was reported related to the knowledge of implant in educational and occupational groups $(\mathrm{p}<0.05)$. The knowledge score was higher in the professionals and highly educated participants. Similar results were observed in the study conducted by Ceyda et al. ${ }^{18}$, and Chowdhary et $a l .{ }^{16}$, in which they found that high level of education coupled with a reasonably higher income have a positive influence on the knowledge.

This survey showed that about $19.6 \%$ of the patients have had implant therapy in the past. There was a significant difference in gender where, $12.6 \%$ of the male and $7 \%$ of the female patients have received implant therapy for 
replacement of their missing teeth. Young adults and high educational group participants (10.2\%) received significantly more implant therapy as related to the other comparable groups. Out of 132 participants who received dental implants, $66 \%$ were satisfied, $26 \%$ were partially satisfied and $8 \%$ were unsatisfied with the therapy. A similar result was observed in studies conducted by Moghadam et al. ${ }^{19}$, and Al- Radha et al. ${ }^{20}$, which showed $85-96 \%$ and $86 \%$ satisfaction raterespectively.

In response to a question about the limitation to receive implant therapy, the high cost of the implant was opted by the majority (41\%) of the participants, followed by long treatment time (15.6\%) and lack of knowledge (13.5\%). A study conducted by Ceyda $e t$ $a l .{ }^{18}$, in Turkish population concluded high coast of the treatment as the strongest argument factor for not choosing implant therapy. A study by Sulieman et $a l^{10}$, found fear as a major barrier against implant therapy. When participants were asked about the right person to place a dental implant, the majority (51.6\%) of them believed that it should be done by specialists. Among the entire respondent, 73\% believed that dental implant treatment should be covered under insurance. A study conducted by Chowdhary et al. ${ }^{16}$, found in their study that, $96 \%$ of the participants were in favor of insurance of dental implant. Male patients favor of insurance coverage for the dental implant more compared to the female patients. Almost three fourth of the studied populations were willing to accept dental implant as a treatment option in the future. In addition patients with young age and high education group showed more interest in receiving the implant therapy in the future

\section{CONCLUSIONS}

Within the limits of the present study, it can be established that population from the Southern region of Saudi Arabia has medium/moderate knowledge about the dental implant treatment. Dentists were found to be the most important source of information to the patients, thus indicating the importance of dentists in spreading the information and give counseling to the patients regarding dental implant treatment. The high charge for implant treatment is one of major barriers for not choosing dental implant therapy. To overcome this barrier, dental implant should be covered under the insurance company so that the majority of the population may get benefit out of this advanced treatment option. Spreading awareness in the general population regarding dental implants can help in abolishing any negative perception of the treatment that may have been due to inadequate knowledge and information.

\section{ACKNOWLEDGMENT}

Nil

\section{CONFLICT OF INTEREST}

Nil

\section{SOURCE OF FUNDING}

Nil

\section{REFERENCES}

1. Masters DH, Tatum H Jr. Dental implants. J Am Dent Assoc 1995;126:1210.

2. Lindh T, Gunne J, Tillberg A, Molin M. A metaanalysis of implants in partial edentulism. Clin Oral Implants Res 1998; 9:80-90.

3. Berge TI. Public awareness, information sources, and evaluation of oral implant treatment in Norway. Clin Oral Implants Res 2000: 11;401-408.

4. Albrektsson, T, Zarb G, Worthington P. Eriksson AR. The long-term efficacy of currently used dental implants: a review and proposed criteria of success. Int J Oral Maxillofac Implants 1986: 1;11-25.

5. Nearby B, Bagewitz IC, Soderfeldt B. Factors explaining the desire for dental implant therapy: Analysis of the results from a longitudinal study. Int $\mathrm{J}$ Prosthodont 2011; 24:437-444.

6. Nearby B, Kronström M, Söderfeldt B, Palmqvist $S$. Changes in attitudes toward a desire for implant treatment: A longitudinal study of a middle-aged and older Swedish population. Int J Prosthodont 2008; 21:481-485.

7. Grogono AL, Lancaster DM, Finger IM. Dental implants: a survey of patients' attitudes. J Prosthet Dent 1989: 62;573-576.

8. Al-Hamdan K. Patients' satisfaction with dental implants in Riyadh, Saudi Arabia. Saudi Dent J 2007: 19;6-11.

9. Zimmer CM, Zimmer WM, William J, Liesener J. public awareness and acceptance of dental implants. Int J Oral Maxillofac Implants 1992; 7:228-232. 
10.Al-Johany S, Al Zoman HA, Al Juhaini M, Al Refeai M. Dental patients' awareness and knowledge in using dental implants as an option in replacing missing teeth: A survey in Riyadh, Saudi Arabia. Saudi Dent J 2010: 22;183-188.

11.Kohli S, Bhatia S, Kaur A, Rathakrishnan T. Public knowledge and acceptance of dental implant treatment in Malaysian Population. J Interdiscip Dent 2014; 4:76-80.

12. Shahabe S, Weam I, Ali O, Mohammed A, Hamoud A, Ali A. Exploring the Knowledge, Attitude and Practice Regarding Hepatitis B Infection Among Dental Students in Saudi Arabia: A Cross-Sectional Study. Open Access Maced J Med Sci. 2019; 7:805-809

13. Salonen MA. Assessment of states of dentures and interest in implant-retained prosthetic treatment in 55year-old edentulous Finns. Community Dent Oral Epidemiol 1994; 22:130-135.

14.Best HA. Awareness and needs of dental implants by patients in New South Wales. Aust. Prosthodont J 1993;7:9-12.

15.Guyatt GH, Cook DJ. Health status, quality of life, and the individual. JAMA 1994; 272:630-631.

16.Pommer B, Zechner W, Watzak G. Progress and trends in patients' mindset on dental implants. I: Level of information, sources of information and the need for patient information. Clin Oral Implants Res 2011; 22:223-229.

17. Chowdhary R, Mankani N, Chandraker NK. Awareness of dental implants as a treatment choice in urban Indian populations. Int $\mathbf{J}$ Oral Maxillofac Implants 2010; 25:305-308.

18. Tepper G, Haas R, Mailath G. Representative marketing-oriented study on implants in the Austrian population. I. Level of information, sources of information and need for patient information. Clin Oral Implants Res 2003; 14:621-633.

19.Ceyda ÖT, Zeynep ÖK, Kemal Ş. Patients' knowledge and awareness of dental implants in a Turkish subpopulation. J Adv Prosthodont 2014; 6:133-137.

20.Moghadam M, Dias R, Kuyinu E, Ferguson MB, Mucciolo T, Jahangiri L. "Pre-doctoral fixed implant patient satisfaction and challenges of a clinical implant competency." J Dent Educ 2012: 76;437-442.

21.Al-Radha ASD. Impact of Anxiety on the Satisfaction of Dental Implant Patients. J Prosthodont 2017 Dec 14. [Epub ahead of print]. 